\title{
Crecimiento predestete de terneros Criollo argentinos
}

\author{
Fernando Holgado ${ }^{3^{*}}$ (1) M Maria Florencia Ortega ${ }^{2} \bowtie$ (1) Alicia E. Rabasa ${ }^{3} \bowtie$ (1) Gabriela E. Cantarella $a^{3}$ (1)
}

Asociación Argentina de Criadores de Ganado Bovino Criollo, Argentina

\section{Pre-weaning growth of A rgentine Criollo calves}

\begin{abstract}
A bstract. The weaning weight of calves depends mainly on the preweaning growth rate, and it is associated with the dairy ability of the cow. Milk is the main food available during the first two months of life of the calf and it is from the third month when the consumption of forage begins to take relevance, being of importance its quality. In the N orthwest of Argentina, bovine breeding is based on the use of C4 perennial grasses with summer growth, high levels of fiber and moderate digestibility. The objective of this work was to evaluate the weight gain of Creole calves in two stages prior to weaning: from birth to 130 days of life and from 131 days to weaning at 205 days. We worked with the offspring born in 2014, 2015 and 2016. For the analysis we used proc GLM from the statistical package SAS. The model included as fixed effects the mother's age at birth, the sex of the calf, and the year of delivery. The results indicate significant effects for the three variables analyzed $(P<0.05)$. The average weight gain from birth to 130 days of life was $648.0 \mathrm{~g} / \mathrm{d}$, being $422.8 \mathrm{~g} / \mathrm{d}$ higher than in the second period. Male calves presented higher weight gains than females and adult cow calves, 7 to 10 years old at parturition, had the highest values of weight gains in both stages. The daily weight gain, in the second period, represented the $68 \%$ of the weight gain of the first stage, which reflects a decrease in the milk production of the cow that does not it becomes compensated by the available forage.
\end{abstract}

Keywords: Genetics resources, creole breeds, weight gain, beef cattle.

Resumen. El peso al destete de los terneros depende principalmente de la tasa de crecimiento predestete y está asociada a la aptitud lechera de la vaca. La leche es el principal alimento disponible durante los dos primeros meses de vida del ternero y es partir del tercer mes cuando el consumo de forraje empieza a tomar relevancia, siendo de importancia su calidad. En el Noroeste Argentino la cría bovina se basa en la utilización de gramíneas perennes C4 de crecimiento estival, niveles elevados de fibra y moderada digestibilidad. El objetivo del presente trabajo fue evaluar la ganancia de peso de terneros Criollos en dos etapas previas al destete: desde el nacimiento hasta los 130 días de vida y desde los 131 días hasta el destete a los 205 días. Se trabajó con las crías nacidas los años 2014, 2015 y 2016. Para el análisis se utilizó proc GLM del paquete estadístico SAS. El modelo incluyó como efectos fijos la edad al parto de la madre, el sexo de la cría y el año de parto. Los resultados indican efectos significativos para las tres variables analizadas $(P<0.05)$. La ganancia de peso promedio desde el nacimiento hasta los 130 días de vida fue de $648.0 \mathrm{~g} / \mathrm{d}$, resultando superior a las del segundo período de $422.8 \mathrm{~g} / \mathrm{d}$. Los terneros machos presentaron ganancias de peso superiores a las hembras y las crías de vacas adultas, de 7 a 10 años al parto, tuvieron los mayores valores de ganancias de peso en ambas etapas. La ganancia diaria de peso, en el segundo periodo, representó el 68 \% de la ganancia de peso de la primera etapa, lo que refleja una disminución en la producción de leche de la vaca que no llega a ser compensada por el forraje disponible.

Palabras claves: Recursos genéticos, razas criollas, ganancia de peso, ganado bovino

\section{Introducción}

La raza bovina Criollo Argentino desciende del ganado introducido por los españoles a América a partir de 1493 en adelante. La caracterización de este recurso genético es una actividad importante para definir estrategias de utilización. La vaca Criolla se destaca por su fertilidad y capacidad de cría. En cuanto al crecimiento del ternero al pie de la vaca, este está fuertemente asociado a la aptitud lechera de la madre, la cual varía con la edad de la misma. En general, la producción de leche de la vaca cubre bien los requerimientos del ternero en sus dos primeros meses de vida y es a partir del tercer mes cuando la

Recibido: 2020-06-24. A ceptado: 2020-11-20

${ }^{1}$ A utor para la correspondencia: fernandodholgado@gmail.com

IInstituto de Investigación A nimal del Chaco Semiárido, CIAP, INTA Leales, Tucumán, Argentina.

3Facultad de Agronomía y Zootecnia. Universidad Nacional de Tucumán. Tucumán, Argentina. 
producción láctea comienza a disminuir y el consumo de forraje empieza a cobrar relevancia (Cantet, 1983). A medida que avanza en edad el ternero, la disponibilidad de pastura en cantidad y calidad comienza a jugar un rol significativo en su evolución de peso (Cantet, 1983; Morales et al., 2019).

En el Noroeste Argentino (NOA) la alimentación del rodeo de cría se basa en la utilización de pasturas megatérmicas caracterizadas por presentar elevados niveles de fibra detergente neutra. La baja digestibilidad de estas gramíneas, así como la pérdida de calidad con el avance de su estado fenológico y aumento de biomasa, se debe a la gran acumulación de estructuras fibrosas. Esto constituye un factor limitante para el consumo animal e influye en la tasa de crecimiento del ternero (Wilson, 1997; Cardoso, 2013; Valente et al., 2011).

Además, el crecimiento predestete del ternero varía de acuerdo a la raza, edad de la madre, sexo y año de nacimiento, entre otras variables (Cantet, 1983; Johnson et al., 2018). Guitou (1983) estudió diversos efectos sobre diferentes razas bovinas y sus cruzas; incluido el rodeo de bovinos Criollo Argentino de INTA Leales (Tucumán). Para esta raza, el autor indica que los terneros machos, hijos de vacas adultas, alcanzan ganancias de peso desde nacimiento a destete de $713.8 \mathrm{~g} / \mathrm{d}$, llegando al destete con un peso vivo de $175.10 \mathrm{~kg}$, y que los machos son un $7 \%$ más pesados que las hembras. Asimismo, sostiene que la ganancia de peso en los terneros es afectada por la edad de la vaca al parto a través de una relación cuadrática, que asciende desde los 3 años de edad de la madre hasta alcanzar una meseta entre los 7-10 años, para luego descender nuevamente.

El objetivo del presente trabajo fue evaluar el crecimiento predestete de terneros Criollo Argentino considerando dos etapas previas al destete.

\section{M ateriales y M étodos}

La información utilizada fue obtenida del rodeo de ganado bovino Criollo Argentino (BCA) existente en el Instituto de Investigación A nimal del Chaco Semiárido (IIACS) del Instituto Nacional de Tecnología Agropecuaria (INTA). El IIACS se encuentra ubicado en la provincia de Tucumán, Argentina (27\% 11 'S, $\left.65^{\circ} 14^{\prime} \mathrm{W}, 335 \mathrm{msnm}\right)$. El clima es subtropical subhúmedo con estación seca. El promedio anual de Iluvias es de $973.3 \pm 263.4 \mathrm{~mm} /$ año. El $88.2 \%$ del total de precipitaciones se concentra entre los meses de noviembre y abril. La temperatura media del mes más cálido (enero) es de $25.8^{\circ} \mathrm{C}$, con una máxima media de 32.4 y una mínima media de $19.4{ }^{\circ} \mathrm{C}$. La media más baja corresponde al mes de Julio $\left(12.4^{\circ} \mathrm{C}\right)$, con máxima y mínima media de $20.4{ }^{\circ} \mathrm{C}$ y $4.5^{\circ} \mathrm{C}$ respectivamente. Entre los meses de mayo y septiembre se registran heladas, con un promedio de 16 por año (Holgado y Ortega, 2019). Estas condiciones climáticas permiten que las pasturas megatérmicas tengan un ciclo de crecimiento de seis meses y un periodo de reposo el resto del año. En este sentido, la alimentación de los rodeos de cría se basa fundamentalmente en el aprovechamiento de las pasturas por pastoreo directo, en verde y diferido, siendo las especies utilizadas Chloris gayana, Megathyrsus maximus, Brachiaria brizantha y Cynodon dactilon con un rango promedio de producciones acumuladas entre 6000 y $9000 \mathrm{~kg}$ MS/ ha. El módulo de vacas de cría comprende todos los vientres de segundo servicio en adelante y ocupa un total de 97 ha en las que se manejan 97 vacas por año aproximadamente (carga $=1$ cabeza/ ha) con acceso al agua de bebida a través de bebederos. La categoría de vaquillonas de primer servicio es manejada en forma separada (carga = 1.8 cabeza/ ha). El servicio de las hembras, natural a campo, se estaciona en los meses de diciembre, enero y febrero; asignándose un toro cada 25-35 vacas. El criterio de elección del toro para cada lote está basado en el mínimo parentesco entre reproductores con la finalidad de controlar niveles de consanguinidad en la descendencia. La palpación rectal para verificar preñez se realiza a los dos meses de retirados los toros de servicio y se elimina toda vaca que resulte vacía. Al ingreso y salida de servicio y al destete se registra peso y condición corporal (escala de 5 puntos) de los vientres. Los nacimientos ocurren entre mediados de septiembre y mediados de diciembre de cada año, con una concentración en el primer mes (60\%). Los terneros son identificados y pesados dentro de 12 a 24 horas de nacidos, utilizando una balanza digital portátil. La pesada a los 130 días y al destete se realizó con una báscula fija y sin desbaste previo. El destete se realiza hacia fines de abril, con una edad aproximada de 205 días. Las prácticas sanitarias en vacas y terneros comprenden vacunaciones obligatorias y desparasitaciones externas de acuerdo a carga parasitaria. En vacas de cría y en terneros al pie, no se aplican tratamientos antiparasitarios para gastrointestinales. Los lotes son recorridos diariamente por personal capacitado. Los cuidados de los animales 
responden a las recomendaciones establecidas en las guías para el cuidado y uso de animales para investigación.

Para este trabajo se empleó información correspondiente a los nacimientos ocurridos los años 2014, 2015 y 2016. Coincidentemente, durante estos años de evaluación el primer servicio de la categoría vaquillona se realizó a los 15 meses de edad con el objetivo de caracterizar la precocidad sexual de la raza. Por este motivo, ingresaron a servicio vaquillonas de pesos muy variables (200-280 $\mathrm{kg}$ ) y con el fin de no afectar su crecimiento futuro, el segundo servicio se realizó a los 3 años (39 meses) sin cría al pie. Para el análisis estadístico se utilizó proc GLM (SAS, 2004). El modelo incluyó como efectos fijos la edad al parto de la madre (EM), el sexo de la cría (SC) y el año de parto (AP). La edad de la madre presentó 8 categorías: 2, 4, 5, 6, 7 a 10, 11-12, 13-14 y 15-16 años. Se analizaron las ganancias diarias de peso de los terneros desde su nacimiento hasta los 130 días de vida (GMP1) y desde los 131 días hasta el destete (GMP2).

\section{Resultados}

La edad de madre, sexo y año de parición afectaron de manera significativa $(P<0.05)$ tanto la GMP1, como la GMP2 de los terneros. Estos efectos sobre la ganancia de peso predestete y el peso de los terneros al destete se encuentran ampliamente documentados en la bibliografía (Cantet, 1983; Martínez-González et al., 2011; Alves et al., 2015). En el caso de GMP1, el modelo explicó el $55 \%$ de la variación observada. La media general fue $648 \pm 122 \mathrm{~g} / \mathrm{d}$ (CV $19 \%$ ). En el caso de GMP2, el modelo explicó el $34 \%$ de la variación observada. La media general fue $443 \pm 133 \mathrm{~g} / \mathrm{d}$ (CV 31 $\%)$. La GMP2 representó el $68.4 \%$ de la ganancia de peso lograda en primer periodo (cuadro 1).

Cuadro 1. Ganancias diarias de peso predestete (GMP1 y GMP2) y relación entre ambas ganancias (\%) para diferentes edades de vaca.

\begin{tabular}{ccccc}
\hline $\begin{array}{c}\text { Edad de vaca } \\
\text { (años) }\end{array}$ & Número de terneros & $\begin{array}{c}\text { GMP1 } \\
(\mathrm{g} / \mathrm{d})\end{array}$ & $\begin{array}{c}\text { GMP2 } \\
(\mathrm{g} / \mathrm{d})\end{array}$ & (GMP2/ GMP1)*100 \\
\hline 2 & 46 & $379.5^{\mathrm{c}}$ & $335.9^{\mathrm{c}}$ & 88.5 \\
4 & 41 & $631.1^{\mathrm{b}}$ & $418.5^{\mathrm{cb}}$ & 66.3 \\
5 & 42 & $704.9^{\mathrm{ab}}$ & $449.2^{\mathrm{a}}$ & 63.7 \\
6 & 36 & $723.5^{\mathrm{a}}$ & $435.7^{\mathrm{ba}}$ & 60.2 \\
$7-10$ & 56 & $748.6^{\mathrm{a}}$ & $484.7^{\mathrm{a}}$ & 64.7 \\
$11-12$ & 19 & $712.8^{\mathrm{a}}$ & $469.1^{\mathrm{a}}$ & 65.8 \\
$13-14$ & 15 & $698.4^{\mathrm{ab}}$ & $435.8^{\mathrm{ba}}$ & 62.4 \\
$15-16$ & 6 & $680.2^{\mathrm{ab}}$ & $378.0^{\mathrm{cb}}$ & 55.6 \\
\hline
\end{tabular}

a, bDiferentes letras entre filas indican diferencia estadística $(P<0.05)$

El valor máximo de GMP1 correspondió a las crías de vacas de edades entre 7 y 10 años $(748.6 \mathrm{~g} / \mathrm{d}$ ), pero no presentó diferencia significativa respecto a otras edades de madre, con excepción de los vientres de 4 años $(631.1 \mathrm{~g} / \mathrm{d}$ ) y de 2 años $(379.5 \mathrm{~g} / \mathrm{d}$ ) al parto. En función de las ganancias de peso presentadas, las vacas de 2 años al parto lograrían al destete un ternero $59 \mathrm{~kg}$ más liviano que el de vacas de 7 a 10 años. Para el mismo rodeo experimental, Guitou (1983) reportó una diferencia de $22.7 \mathrm{~kg}$ en el peso al destete de los terneros cuando comparó vacas Criollas de 3 años al primer parto con vacas de 7 a 10 años. Esto estaría indicando que la edad al primer parto, 2 o 3 años, afecta de manera importante la tasa de crecimiento y peso al destete de los terneros. La tasa de crecimiento de las crías de vacas de 2 años al canzó solamente el 50 $\%$ de la obtenida por aquellas de vacas de 7 a 10 años de edad. Esto puede ser atribuido a la baja aptitud lechera de la vaquillona con servicio a los 15 meses de edad (Holgado et al., 2017).
En la raza Aberdeen Angus se indica un peso al nacimiento de terneros de vacas de primer servicio a los 15 meses de $28 \mathrm{~kg}$ y $158.1 \mathrm{~kg}$ al destete, con una ganancia diaria de $590 \mathrm{~g} / \mathrm{d}$ (López Valiente et al. 2018). El valor de ganancia de peso resulta claramente superior al encontrado en este trabajo para igual categoría de vientre, lo que pueda deberse a la existencia de importantes diferencias en la alimentación de las vacas. Los autores señalan que la dieta de los vientres A. Angus estuvo basada en verdeos y silaje de maíz durante el periodo invernal y pasturas perennes templadas y verdeos de verano hasta el destete de los terneros. En el caso de Criollo la alimentación se basó en pasturas megatérmicas diferidas $y$ en crecimiento, sin acceso a suplementación. En el mismo trabajo, se indica que los terneros hijos de vaquillonas con servicio a los 27 meses de edad tuvieron mejores ganancias predestete que los hijos de vaquillonas entoradas a los 15 meses (680 g/ d vs $590 \mathrm{~g} / \mathrm{d}$ ); y que las crías de vacas 
multíparas tuvieron mayores ganancias diarias de peso (790 g) respecto a las mencionadas anteriormente. Lo mismo ocurre en la raza Criolla, aunque con magnitudes son considerablemente menores (ganancias de $652 \mathrm{~g} / \mathrm{d}$ en vacas de 7-10 años y $364 \mathrm{~g} / \mathrm{d}$ en vacas de 2 años, para ambos periodos) a las reportadas por López Valiente et al . 2018.

Es importante destacar que las crías de vacas de 1112 años y 13-14 años tuvieron ganancias diarias de peso (GMP1) que no difieren significativamente del alcanzado por las vacas de 7-10 años, lo que manifiesta de cierta manera refleja la longevidad del ganado criollo.

Independientemente de la edad de madre, la GMP2 resultó en promedio equivalente al $68 \%$ de la lograda en el primer periodo (GMP1). Esto se relaciona con el aporte nutricional de la leche y el pasto en cada periodo. La influencia de la producción de leche en el crecimiento del ternero disminuye a partir del tercer mes de vida, haciéndose prácticamente independiente al quinto mes y cobrando relevancia la pastura (Cantet, 1983; Morales et al., 2019). Al analizar la relación GMP2/ GMP1 para las diferentes edades de vacas se observa que la vaca de primer parto mantiene el $85 \%$ de la lograda en GPM 1. Esto indicaría que su producción de leche es baja desde el inicio de la curva de lactancia y se mantiene baja a través del tiempo. Mientras en las otras edades, se observa en general que la vaca Criolla cría bien a su ternero en los primeros 130 días, manteniendo una ganancia diaria de peso del orden de los $700 \mathrm{~g} / \mathrm{d}$, y que a partir de allí la tasa de ganancia de peso cae al $65 \%$. Esto indica un menor aporte lácteo en el segundo periodo, que no llega a ser compensado por el mayor consumo de pasturas megatérmicas, para sostener el ritmo de crecimiento inicial, debido a su moderada calidad forrajera. Como lo demuestra Holgado (2002), terneros destetados a los 4 meses y alimentados exclusivamente con pastura megatérmicas tuvieron pérdidas de peso del orden de -0.062 y $-0.077 \mathrm{~kg} / \mathrm{d}$, en hembras y machos respectivamente. Mientras que aquellos que no fueron destetados tuvieron ganancias de 0.483 $\mathrm{kg} / \mathrm{d}$ las hembras y $0.567 \mathrm{~kg} / \mathrm{d}$ los machos. Es decir que el aporte de leche, aunque sea cuantitativamente bajo, actúa como un suplemento nutricional de gran importancia estratégica para mantener ganancia de peso. En otro trabajo, Holgado et al., 2019, evaluaron el efecto de la suplementación al pie de la madre, en la raza Criolla, sobre la ganancia de peso predestete de terneros machos de 100 días de edad. La suplementación duró 107 días y la ganancia de peso del testigo se incrementó de $585 \mathrm{~g} / \mathrm{d}$ a $1.024 \mathrm{~g} / \mathrm{d}$ cuando recibió ración a voluntad. Esto permite apreciar como la leche y el pasto lejos están de posibilitar la expresión del potencial de crecimiento de la raza. La alta respuesta obtenida, en términos de ganancia de peso y eficiencia aparente de conversión (4.26) sería reflejo de un bajo aporte de leche y moderada calidad pasturas megatérmicas.

En el cuadro 2 se muestra el efecto del año sobre las GMP1 y GMP2. A mbas variables son afectadas por el año de parición. Año representa un efecto al azar, que afecta la ganancia y el peso al destete de los terneros y aparece reflejado en numerosos trabajos de investigación (Guitou, 1983; Cantet, 1983; MartínezGonzález et al., 2011; Alves et al., 2015). El efecto del año de nacimiento sobre las GMP1 puede ser consecuencia del estado corporal de la vaca durante la lactancia. Holgado y Rabasa (1998) evaluaron el efecto de la condición corporal del vientre sobre el peso al destete de sus crías. Concluyen que el estado de la vaca durante la lactancia se relaciona positivamente con el peso al destete de las crías; siendo este efecto seguramente mediado por la producción de leche de la vaca. En el caso de la GMP2, esta reflejaría en primer lugar variaciones en la cantidad y calidad del forraje disponible para el ternero; y en segundo lugar el aporte de leche materna, el cual pierde relevancia en la medida que la curva de lactancia avanza.

Cuadro 2. Ganancias diarias de peso predestete (GMP1 y GMP2) para diferentes años de parición y relación entre periodos (GMP2/ GMP1).

\begin{tabular}{ccccc}
\hline Año & $\begin{array}{c}\text { Número de } \\
\text { terneros }\end{array}$ & $\begin{array}{c}\text { GMP1 } \\
(\mathrm{g} / \mathrm{d})\end{array}$ & $\begin{array}{l}\text { GMP2 } \\
(\mathrm{g} / \mathrm{d})\end{array}$ & GMP2/ GMP1 \\
\hline 2016 & 80 & $609.3^{\mathrm{b}}$ & $520.9^{\mathrm{a}}$ & 85.5 \\
2015 & 85 & $675.7^{\mathrm{a}}$ & $431.4^{\mathrm{b}}$ & 63.8 \\
2014 & 96 & $694.6^{\mathrm{a}}$ & $333.5^{\mathrm{c}}$ & 48.1 \\
\hline
\end{tabular}

a, b, cDiferentes letras entre filas indican diferencia estadística $(P<0.05)$

Ferrando et al. (2006) analizan 11 años de resultados correspondientes a un sistema de cría integrado por ganado Criollo Argentino y Aberdeen Angus. Al considerar la ganancia de peso predestete y el peso de destete de la raza Criolla, encuentran valores medios de $0.696 \mathrm{~kg} / \mathrm{d}$ y $152.3 \mathrm{~kg}$ respectivamente. Con variaciones que fueron desde mínimos de $0.634 \mathrm{~kg} / \mathrm{d}$ y 141.2 kg, y máximos de 0.815 kg/ d y 173.8 kg. Estos 
resultados muestran la magnitud e importancia del año en la tasa de crecimiento y peso al destete del ternero.
En el cuadro 3 se muestran las GMP1 Y GMP2 correspondientes a terneros machos y hembras. El efecto sexo afectó de manera significativa a ambas variables $(P<0.05)$.

Cuadro 3. Ganancias de peso de acuerdo al sexo de los terneros para los periodos GMP1 y GM P2 y su relación

\begin{tabular}{lcccc}
\hline Sexo & $\begin{array}{c}\text { Número } \\
\text { de terneros }\end{array}$ & $\begin{array}{r}\text { GMP1 } \\
(\mathrm{g} / \mathrm{d})\end{array}$ & $\begin{array}{c}\text { GMP2 } \\
(\mathrm{g} / \mathrm{d})\end{array}$ & GMP2/ GMP1 \\
\hline Macho & 126 & $694.1^{\mathrm{a}}$ & $444.2^{\mathrm{a}}$ & 64.0 \\
Hembra & 135 & $625.6^{\mathrm{b}}$ & $407.5^{\mathrm{b}}$ & 65.1 \\
\hline
\end{tabular}

a, b Diferentes letras entre filas indican diferencia estadística $(P<0.05)$

El aumento de peso de los terneros machos durante los dos periodos evaluados fue un $11 \%$ (GMP1) y $9 \%$ (GMP2) superior al de las hembras (Cuadro 3). Estos valores coinciden con lo descripto por Holgado et al. (2016), con resultados de ganancia promedio de peso predestete de 755 y $664 \mathrm{~g} / \mathrm{d}$ para terneros machos y hembras, hijos de vacas de 5 a 11 años de edad. El efecto del sexo está ampliamente documentado (Cantet, 1983; Guitou, 1983; Martínez-González et al., 2011; A lves et al ., 2015)

\section{Conclusión}

Las ganancias de peso en terneros de raza Criollo Argentino, en los dos periodos evaluados: GMP1 y GMP2, fueron influenciadas por la edad de la madre, sexo del ternero y año de parición. El aumento diario de peso del segundo periodo fue equivalente al $68 \%$ del obtenido en los primeros 130 días. Esta caída de la tasa de crecimiento del ternero sería reflejo de la disminución del aporte de leche materna en la alimentación del ternero y el incremento del forraje de moderada calidad que debe cubrir esta disminución. Las ganancias de peso del primer periodo muestran que la raza Criolla Argentina presenta una moderada aptitud lechera, adecuada para criar bien su ternero. No es el caso de las vacas de 2 años al primer parto, donde el crecimiento del ternero fue relativamente bajo.

\section{Literatura Citada}

Alves, F.V., Brito, M.C.B., Juliano, R.S., A breu, U.G.P., Souza, J.C. y Santos S.A. 2015. Fatores que influenciam no desempenho de bezerros Pantaneiros Criados em pastagen nativa no pantanal. Actas iberoamericanas de Conservación A nimal. AICA 5: 38-43.

Cantet R.J.C.1983. El crecimiento del ternero. 81 pag. Editorial Hemisferio Sur. Argentina.

Cardoso S. 2013. Acúmulo de massa seca verde e características anatômicas, químicas e biológicas das lâminas foliares de capimmassai sob doses de calcário e nitrogênio. Ph.D. Teses. UFGD, Dourados MS, Brazil. 91 p.

Ferrando, C., Paloma, E., Namur, P., y Leguiza, D. 2006. Ganado bovino Criollo Argentino y Aberdeen Angus en Los Llanos de La Rioja: Resultados de 11 años de evaluación en sistema de cría. Ediciones INTA, EEA La Rioja. ISSN 1669-323X

Guitou, H. R. 1983. Evaluation of crossbreeding in beef cattle in Tucumán, Argentina. Tesis de Master of Science. Iowa State University. A mes, Iowa, EEUU.

Holgado, F.D. y Rabasa de Sal Paz, A. 1998. Relación entre condición corporal de la vaca al destete y los pesos al nacer y destete de la futura progenie. Rev. Arg. Prod. Anim. vol. 18: 302-303.
Holgado, F.D. 2002. Ganancia de peso de terneros destetados a los 4 meses de edad, alimentados con pastura tropical. Rev. Arg. Prod. A nim. 22: 81

Holgado, F.; Martínez B.; Díaz, A.; Soraire J. 2016. Influencia del fenotipo de la madre en el peso al nacimiento y ganancia de peso en terneros Criollos Argentino. AICA 8 (2016) 13-15.

Holgado, F.; Fernández, J.L., Ortega M.F. 2017. Influencia del peso de servicio a los 15 meses sobre el peso al nacer y ganancia predestete de las crías en Criollo Argentino. AICA 10 (2017) 57-62.

Holgado, F.D. y M.F. Ortega. 2019. Caracterización productiva del bovino Criollo Argentino: periodo 2006-2016. Ediciones INTA, Colección Investigación, Desarrollo e Innovación. Buenos Aires, Argentina. ISBN 978-987-521-987-8.

Holgado, F.D., Ortega, M.F., Cantarella, G., y Martínez, R. 2019. Efecto de la suplementación al pie de la madre sobre el peso de terneros de la raza Criollo Argentino. Comunicación, Rev. Arg. Prod. Anim. 39, supl 1: 46.

Johnson, K.F.; Chancellor, N.; Burn, C.C., Wathes D.C. 2018. Analysis of pre-weaning feeding policies and other risk factors influencing growth rates in calves on 11 commercial dairy farms. Animal (2018), 12(7):1413-1423. 
López Valiente, S.; Barrionuevo, E.; Canaparo, J.: Maresca, S.; Rodríguez, A.M. y Miccoli, F. 2018. Efecto de la edad de la madre en el crecimiento del ternero hasta el destete y producción de leche. Revista Argentina de Producción A nimal. 38 Supl. 1; 94.

Martínez-González, J.C., Gutiérrez-Michel, J.F., Briones-Encinia, F., Lucero-Magaña, F.A. y CastilloRodríguez, S.P. 2011. Factores no genéticos que afectan el peso al nacer y destete de terneros Angus. Zootecnia Trop., 29 (2): 151-159.

Morales, R. M., Menéndez-Buxadera, A., DemydaPeyrás, S. y Molina, A. 2019. Genetic effects of season on the preweaning growth of beef cattle: $A$ first approach to Retinta calves. Revista Colombiana de Ciencias Pecuarias. 33 (2), 134 - 143. https:/ / doi.org/ 10.17533/ udea.rccp.v33n2a0
SAS Institute Inc. 2004. SAS Online 9.1.03 Cary, NC: SAS, Institute Inc.

Valente, T. N. P., E. Detmann, S. C. Valadares Filho, M. Cunha, A. C. Queiroz, C. B. Sampaio. 2011. In situ estimation of indigestible compounds contents in cattle feed and feces using bags made from different textiles. Braz. J. Anim. Sci. 40:666-675. https:/ / doi.org/ 10.1590/ S1516-35982011000300027

Wilson, J.R. 1997. Structural and anatomical traits of forages influencing their nutritive value for ruminants. Simposio internacional sobre produçã animal em pastejo. Viçosa, MG. Anais Viçosa, MG: UFV. pp.173-208. 